\title{
BARYON STRUCTURE*
}

\author{
M. RADICI \\ Istituto Nazionale di Fisica Nucleare, Sezione di Pavia, and \\ Dipartimento di Fisica Nucleare e Teorica, Università di Pavia, \\ via Bassi 6, \\ 27100 Pavia, Italy \\ E-mail: radici@pv.infn.it
}

\begin{abstract}
A review of the theoretical activity in Italy in the research field of Hadronic Physics is given. Specific focus is put on phenomenological models based on the effective degrees of freedom of constituent quarks, on parton distributions in hard processes in the Bjorken limit and on the possibility of linking the two concepts via evolution equations. A brief introduction is given also about the socalled generalized parton distributions.
\end{abstract}

\section{Introduction}

The Quantum Chromodynamics (QCD) is the commonly accepted theory of strong interactions of elementary particles. It displays two fundamental properties: the asymptotic freedom at very short distances and the confinement of quarks and gluons inside the observed colorless hadrons. While the former allows for spectacular precision tests of the theory in the framework of the socalled perturbative QCD (pQCD), the latter yet cannot be explained from first principles. The reason is that the confinement happens at the hadronic scale (typically, the nucleon size) where the running coupling, $\alpha_{s}$, is so large to forbid any perturbative approach. There are several ways to attack the problem: formulating QCD sum rules, solving the theory on the lattice, etc.. Here, I will focus on the attempts to explore the nonperturbative regime using ansätze induced from the fundamental symmetries of QCD itself. More explicitly, this kind of activity, conventionally included in the socalled Hadronic Physics, can be reformulated as the study of confined systems of strongly interacting quarks and gluons. From this point of view, the overlap with the know-how of the Nuclear

*This work is partly supported by COFINANZIAMENTO MURST-PRIN-01 
Physics community is very large, since the hadron can be considered as a strongly correlated many-body system.

In the following, I will review the theoretical contributions in Italy to this research field. In Sec. 2 I will focus on different formulations of lowenergy phenomenological models based on the effective degrees of freedom of constituent quarks, with emphasys on the covariance and gauge invariance of such models. In Sec. 3 I will concentrate on the analysis of hard processes at a higher scale, in a typical regime of Deep-Inelastic Scattering (DIS), with the aim of exploring the (spin) properties of partons inside the hadrons and, possibly, of establishing a link between the concept of constituent quark (around $\Lambda_{\mathrm{QCD}}$ ) and current quark (in the DIS regime) through the evolution equations. Finally, in Sec. 4, I will consider the socalled Generalized Parton Distributions (GPD), a very promising formalism which unifies both exclusive and (semi)inclusive hard processes, as it may offer a gauge invariant solution to the puzzle of the nucleon spin decomposition into the spins of its elementary constituents.

\section{Constituent Quark Models}

Constituent Quark Models (CQM) are described by hamiltonians involving effective degrees of freedom, the constituent quarks, which are massive quasi-particles moving in a background field generated by gluons. Confinement is realized by a long-range phenomenological potential $V_{\text {conf }}$, while the short-range interaction $V_{\mathrm{qq}}$ is treated perturbatively. The spectrum is obtained by diagonalizing the hamiltonian. Since this is not sufficient to discriminate among different dynamical models, hadron electromagnetic observables are also deduced by computing matrix elements of the electromagnetic current operator between the obtained eigenfunctions.

A typical example is represented by the socalled hypercentral CQM of baryons, where the unperturbed hamiltonian is given by a linear and a coulombic contributions in terms of the hyperradius $x=\sqrt{\rho^{2}+\lambda^{2}}$, with $\rho, \lambda$, the usual Jacobi coordinates of the three-quark system. Such a threebody hypercentral potential satisfactorily reproduces the SU(6) gross structure of the light-baryon spectrum using just two free parameters. The further hyperfine splittings were interpreted, in a first version of the model, by adopting for $V_{\mathrm{qq}}$ the spin-spin part of the hyperfine interaction induced by a one-gluon-exchange (OGE) mechanism ${ }^{1}$ (employing two new parameters). However, despite the introduction of relativistic corrections both of kinematical and dynamical type to the current operator ${ }^{2}$, several drawbacks 
arise. In particular, the wrong ordering in the spectrum of the Roper resonance $J^{P}=\frac{1}{2}^{-}$with respect to its positive-parity partner, and the impossibility of finding a consistent parametrization for the root-mean-squared (rms) charge radius $\left\langle r^{2}\right\rangle_{\mathrm{ch}}$ and the nucleon (N) electromagnetic form factors at the same time. A new version of the hypercentral CQM has been recently released ${ }^{3}$, which implements $V_{\mathrm{OGE}}$ by adding also an explicit isospin dependence (and introducing four new parameters), as suggested also by other models of $V_{\mathrm{qq}}$ (see Sec.2.2 in the following). This $\mathrm{SU}(6)$-breaking feature is crucial to get a much more accurate description of the spectrum.

However, the hypercentral CQM is based on a nonrelativistic description, which is known to be inadequate for constituent quarks that are essentially relativistic particles. The question arises why these kinds of nonrelativistic CQM reproduce several hadron properties at the quantitative level. A possible answer stems from the socalled General Parametrization (GP) method, according to which the (QCD) exact matrix element of an hermitean operator can be represented by a linear parametrization of the complete set of spin-flavor operators of three constituent quarks. The key observation is that there is a hierarchy in the size of the parameters, the nonrelativistic CQM retaining just the first largest ones. Several applications of the GP method lead to interesting results ${ }^{4,5,6,7}$. The GP method considers matrix elements in the hadron rest frame, thus loosing the property of covariance but keeping Lorentz invariance.

Another series of works consists in covariant approaches based on a given hamiltonian with a finite number of degrees of freedom. Along this line of investigation, a relativistic quantum-mechanical formulation is only possible in terms of unitary representations of the Poincaré group. There are at least three unitarily equivalent ways to do it: the instant form (IF), the light-front form ( $\mathrm{LF}$ ) and the point form (PF) of dynamics. They can be distinguished by looking at the different ways of implementing the interaction into a free covariant theory while keeping the covariance and the Lie algebra of the 10 Poincaré generators, the socalled Bakamjian-Thomas (BT) construction. The instant form is the usual context in which all relativistic field theories, including QCD, are developed. In the following, I will review results obtained by the LF and PF descriptions of relativistic CQM, respectively. 


\subsection{Front Form Description}

The starting point is the eigenvalue problem for a Poincaré- $(\mathcal{P}$ - $)$ covariant mass operator $M_{0}+V$, with $M_{0}$ the free mass operator. If the interaction $V$ is independent of the total momentum $\mathbf{P}_{\text {tot }}$ of the system and commutes with the LF spin operator, then the eigenvalue problem can be rewritten in terms of an intrinsic relativistic kinetic operator (involving the $N$ constituent momenta $\mathbf{k}_{i}$ such that $\sum_{i}^{N} \mathbf{k}_{i}=0$ ) and of the potential $\mathcal{V}=\mathcal{R} V \mathcal{R}^{\dagger}$, where $\mathcal{R}$ is the Melosh rotation connecting the spins in the (IF) standard representation to the LF representation. When calculating electromagnetic observables, the current operator $J^{\mu}$ must be a conserved current and must fulfill the socalled extended $\mathcal{P}$ covariance, i.e. it must be $\mathcal{P}$-covariant and it must respect parity $(P)$ and time-reversal $(T)$ invariance. Then, the form factors can be expressed in terms of matrix elements of only $J^{+}$and the socalled angular condition is satisfied, i.e. a relation between the current matrix elements must hold such that the number of form factors corresponds to the number of $J^{+}$independent matrix elements. If, moreover, the Drell-Yan condition for the momentum transfer is satisfied, i.e. $q^{+}=0$, the momentum conservation on the light-cone implies, for systems with total spin $J \leq \frac{1}{2}$, a suppression of many-body contributions to the electromagnetic vertex, corresponding, in field theory, to Feynman diagrams involving nonvalence $q \bar{q}$ pairs. Thus, it is legitimate to use the Impulse Approximation (IA) for the current operator $J_{\mathrm{IA}}^{\mu}$. Introducing parametrized form factors for the constituent quarks, a global fit to the spectrum of light baryons and to the $\mathrm{N}$ and $\pi$ elastic form factors is achieved, which constrains the parameters in order to make predictions for the transitions to the various $\mathrm{N}^{*}$ resonances. Using for $\mathcal{V}$ the Capstick-Isgur potential based on a linear confining term and on an SU(6)-breaking effective gluonexchange contribution, which includes a Coulomb-like part, a spin-spin part responsible for the hyperfine splittings, and a tensor part, a successful and consistent picture of light hadrons is obtained ${ }^{8}$.

However, the use of $J_{\mathrm{IA}}^{\mu}$ breaks the angular condition. This is particular evident in the case of the $\mathrm{N} \rightarrow \Delta$ transition, where three form factors (electric, $G_{E}$; magnetic, $G_{M}$; coulombic, $G_{C}$ ) correspond to four independent matrix elements. Usually, three out of the latter ones are chosen to be independent and the fourth one is forced to fulfill the angular condition, but this selection is arbitrary reflecting in very different results for the various multipoles contributing to the transition ${ }^{9}$. In the case of a system with total spin $J \leq \frac{1}{2}$, there is a perfect correspondence between 
form factors and $J_{\text {IA }}^{+}$matrix elements. However, the angular condition is still broken, or, equivalently, the rotational covariance of the current operator is lost. For the example of the $\mathrm{N}$ elastic form factors, the $G_{M}^{\mathrm{N}}\left(Q^{2}\right)$ can be deduced from different components of $J_{\text {IA }}^{\mu}$ obtaining two different results ${ }^{10}$. Moreover, it seems not possible to reproduce at the same time the two main evidences for the breaking of SU(6) symmetry: the nonvanishing neutron electric form factor $G_{E}^{\mathrm{n}}\left(Q^{2}\right)$ and the deviation of the ratio $G_{M}^{\mathrm{p}}\left(Q^{2}\right) / G_{M}^{\mathrm{n}}\left(Q^{2}\right)$ from the value -1.5 . Cardarelli and Simula ${ }^{10}$ suggest a common recipe to these problems. The central idea is that for a system with $J \leq \frac{1}{2}$ in the $q^{+}=0$ frame the induced loss of rotational covariance implies that the $J_{\mathrm{IA}}^{\mu}$ matrix elements depend upon the choice of a null 4vector $\omega$ perpendicular to the LF null plane $x^{+}=0$. The constituent quark momenta can then be reparametrized as to be all on shell, as demanded by the IA. Consequently, the covariant decomposition of the current operator contains new spurious structures explicitly depending on $\omega$. Overcoming the angular condition problem means selecting appropriate components of $J_{\mathrm{IA}}^{\mu}$ such that the calculated observables do not depend upon spurious contributions, as they have to. In this way, good results are obtained for the electromagnetic observables of pion and nucleon, while the situation is less clear in the case of $\mathrm{N}$ inelastic transitions because of the scarce amount of data available ${ }^{11}$.

For spin- 1 systems it is known that also for $q^{+}=0$ the contribution of diagrams involving $q \bar{q}$ pairs does not vanish, so that the question arises if an IA in a LF model with fixed number of degrees of freedom is possible. Melikhov and Simula ${ }^{12}$ show that this is possible by extending the previous analysis to the tensor structure of the deuteron electromagnetic vertex, with the interesting outcome that also "good" components for the initial and final LF polarization vectors of the spin- 1 system must be selected in order to get matrix elements free from spurious contributions.

An alternative solution to the problem of rotational covariance is discussed by Lev, Pace and Salmè ${ }^{9}$. From the fact that LF Lorentz boosts are interaction free the wave function is usually split in a cm and an intrinsic part. Similarly, taking matrix elements of the exact current operator $J^{\mu}$ on projected $\mathrm{N}$ states in the Breit frame with $\mathbf{q} \| \hat{z}$, it is possible to construct a current operator, acting only on the intrinsic Hilbert subspace, that is extended $\mathcal{P}$-covariant, hermitean, conserved, and fulfills charge normalization. For all the properties to hold, the choice of the frame is crucial, because the rotational covariance around $\hat{z}$ is sufficient to grant all the other features. Since in the LF these rotations are interaction free, the current 
operator can be built starting from $J_{\mathrm{IA}}^{\mu}$, while the angular condition is still satisfied. For the example of the deuteron, improvement is obtained for both the magnetic and quadrupole momenta, indicating the importance of relativistic covariance even at $Q^{2}=0$; the three electromagnetic form factors can be unambiguously calculated, even if the results are still affected by model dependence in the choice of the $\mathrm{N}-\mathrm{N}$ interaction and of the $\mathrm{N}$ form factors ${ }^{9}$.

As already stressed, the above recipe of building an "intrinsic" covariant operator relies on the choice of the Breit frame with $\mathbf{q} \| \hat{z}$, or equivalently for $\mathbf{q}_{\perp}=0$. Therefore, the pair diagrams do contribute to one-loop electromagnetic interactions in a covariant field-theoretical approach. In Ref. ${ }^{13}$, Simula discusses the issue of rotational covariance by comparing it with the previous recipe based on the elimination of spurious contributions. By analyzing the form factors of the pion and of the deuteron, he shows that the two approaches are inequivalent, since the LF Lorentz transformation from the $q^{+}=0$ frame to the $\mathbf{q}_{\perp}=0$ one is interaction dependent. The claim is that the pair diagrams can be safely neglected in the $q^{+}=0$ frame, but they are important at $q^{+} \neq 0$ for light hadrons, disappearing in the heavy-quark limit. In another paper ${ }^{14}$, de Melo, Frederico, Pace and Salmè analyze the electromagnetic form factor of a pion-like $q \bar{q}$ bound state by building a covariant model of the Bethe-Salpeter amplitude with a symmetric vertex function in momentum space, deduced from a lagrangian with pseudoscalar pion-quark coupling at the one-loop level. By parametrizing the Breit frame with an angle $\alpha$ indicating the position of the momentum tranfer $\mathbf{q}$ in the $\widehat{x z}$ plane, they study the relative weight of the spectator and pair diagrams with varying $\alpha$ between the boundary positions $\alpha=0$ $\left(q^{+}=0\right)$ and $\alpha=90^{\circ}\left(q^{+}=q\right)$, recovering essentially the same results as in Ref. ${ }^{13}$. However, by rescaling the constituent quark masses to the $\mathrm{N}$ masses such that the pion-like system becomes a weakly relativistic spin-0 system with approximately the deuteron mass, the interesting result emerges that the pair diagram can be safely neglected for $Q^{2} \leq 1$ and for any $\alpha$, i.e. in any Breit frame with $q_{y}=0$.

\subsection{Point Form Description}

In the $\mathrm{PF}$ realization of relativistic quantum mechanics only the fourmomentum operator $P^{\mu}$, generating the space-time translations, contains the interaction. The theory is thus manifestly covariant and the current matrix elements can be accurately calculated in any frame. It is convenient 
to introduce the socalled velocity states, defined in such a way that, under a Lorentz transformation $\Lambda$, the momenta and the spins of the constituents are rotated by the same Wigner rotation $R_{W}$; contrary to the LF formalism, it is then possible to couple orbital angular momenta and spins in the same way as it is done in a nonrelativistic theory. By construction, the velocity states are eigenfunctions of $M_{0}$ and of the free momentum operator $P_{0}^{\mu}=M_{0} V^{\mu}$. Interactions are introduced in the momentum operator as $P^{\mu}=P_{0}^{\mu}+P_{I}^{\mu} \equiv M V^{\mu}$, where the mass operator is $M=M_{0}+V$. The BT construction is realized if $M$ is a scalar (or, equivalently, $V$ is rotationally invariant) and commutes with $V^{\mu}$ (i.e., $V$ is a local interaction); the Lie algebra of the Poincaré group, then, takes the form of the eigenvalue problem $M|\psi\rangle=m|\psi\rangle$ for a system of mass $m$ made up by $N$ constituents.

If the current operator $J^{\mu}$ satisfies the same Lie algebra, it can be shown to be an irreducible tensor operator of the Poincaré group. Then, a generalized Wigner-Eckhart theorem implies that if the matrix elements are taken on the eigenstates of $P^{\mu}$, they can be written as the product of covariant objects (the Clebsh-Gordan coefficients of the Poincaré group) and invariants $G^{\mu}\left(Q^{2}\right)$ (the "PF" form factors). In the Breit frame with q \| $\hat{z}$, the Clebsh-Gordan coefficients become the identity, so that the $G^{\mu}\left(Q^{2}\right)$ can be deduced by directly computing the matrix elements of $J^{\mu}$ in this frame. Recalling the discussion developed in Sec. 2.1, the angular condition is here automatically satisfied if $J^{\mu}$ is also $P$ - and $T$-reversal invariant: for a system with total angular momentum $J$ there are only $2 J+1$ invariant "PF" form factors.

By projecting the initial and final states in Breit frame upon the corresponding velocity states with $v_{i}=-Q / 2 m$ and $v_{f}=Q / 2 m$, it is possible to directly calculate the needed matrix elements of $J^{\mu}$ using the elementary degrees of freedom. As in the LF case, the IA is adopted but the constituents are here considered point-like. The presence of velocity states implies that the momentum conservation constraint cannot be written in close form. In other words, the impulse $q=m v_{f}-m v_{i}$ delivered to the system is different from the one absorbed by the active constituent. For this reason, this IA in PF is called the Point-Form Spectator Approximation (PFSA) ${ }^{15}$. The role played by the velocity dependent boosts is crucial in determining the correct $Q^{2}$ fall-off of the form factors.

In Refs. ${ }^{15,16,17}$ results are shown for the PFSA in a relativistic CQM based on the socalled Goldstone-Boson Exchange (GBE) quark-quark interaction $V_{\mathrm{GBE}}$, which is dictated by the spontaneous breaking of QCD chiral symmetry at low energy. As such, the model is applicable below the 
energy threshold of chiral symmetry restoration, in any case for $Q^{2} \lesssim 3 \div 4$ $(\mathrm{GeV} / c)^{2}$. At variance with $V_{\mathrm{OGE}}, V_{\mathrm{GBE}}$ explicitly depends on flavor; its peculiar structure is responsible for the very good reproduction of the lowlying light and strange baryon spectra using just five parameters. In particular, it offers a natural solution to the problem of the correct position of the Roper resonance energy level. Covariant calculations of electroweak $\mathrm{N}$ form factors have been performed in the PFSA obtaining an overall very consistent picture ${ }^{17}$. The agreement is particularly impressive if it is considered that point-like constituent quarks are used and that only the GBE model wave functions are input into the calculation, i.e. without introducing further adjustable parameters. Comparison with pure nonrelativistic calculations indicates that the relativistic boosts play a crucial role even at $Q^{2}=0$. Moreover, the neutron charge form factor is highly sensitive to the details of the GBE model, since it is mostly given by the small SU(6)breaking components of the GBE eigenfunctions. Only for the magnetic response the results show an evident deviation from data at $Q^{2}>2(\mathrm{GeV} / c)^{2}$ that leaves room for quantitative improvements beyond the PFSA ${ }^{17}$.

\subsection{Baryon Spectrum and Chiral Symmetry Breaking}

Chiral symmetry breaking is one of the fundamental QCD features that must be taken into account when dealing with the low-energy properties of hadrons. In this section, the problem is reconsidered replacing a hamiltonian approach with a phenomenological model based on the Nambu JonaLasinio (NJL) lagrangian. The strong attractive force between quarks in the $J^{P}=0^{+}$channel induces instabilities in the Fock vacuum of massless quarks, breaking the chiral symmetry and generating a dynamical quark mass $m^{*}$ related to the vacuum condensate $\langle\bar{q} q\rangle$ and of the same order of magnitude as the constituent quark mass parameters used in CQM. For increasing density and temperature the model reproduces the transition from the broken phase to the chiral symmetry restoration. In fact, assuming an homogeneous, isotropic quark matter inside the baryon, it is possible to calculate the radial dependence of the quark dynamical mass $m_{a}^{*}(r)$ for the flavor $a$ in a Local-Density Approximation (LDA). It reflects the density change when moving away from the baryon center: inside the baryon the density (and, consequently, the Fermi momentum) is very large, $m_{a}^{*}(r)$ is very small and chiral symmetry is restored; getting through the baryon surface, the density drops while $m_{a}^{*}(r)$ increases until it reaches its constituent quark-like asymptotic value, which is determined by the interaction of the 
quark with the chirally broken vacuum.

The energy of the vacuum is ascribed to the filled negative-energy levels of the Dirac sea, which put a pressure $p_{\text {vac }}$ on the baryon. Viceversa, inside the baryon the pressure $p_{\mathrm{B}, \mathrm{vac}}$ is much smaller since the density is very high. The equilibrium is given by the counterbalancing pressure $p=p_{\mathrm{vac}}-p_{\mathrm{N}, \mathrm{vac}}$ induced by the quarks inside the baryon. This stability condition fixes the size and mass of the baryon ${ }^{18}$. According to different choices of the model parameters, the range $\left\langle r^{2}\right\rangle=0.76 \div 0.80 \mathrm{fm}$ is obtained for the $\mathrm{N}$ radius in very good agreement with the experimental datum ${ }^{18}$. Also the baryon octet spectrum is fairly well reproduced within a $\pm 5 \%$ deviation, even if the assumed degeneracy between $u$ and $d$ flavors prevents from distinguishing the proton from the neutron and other isospin multiplets. The deviation from the baryon decuplet spectrum is, instead, more evident since no spinspin force has been taken into account in the model, while the octet-decuplet splitting is usually attributed to the spin-spin interaction. Introducing an effective perturbation, based on the spin-spin part of the OGE potential, does not substantially improve the situation ${ }^{18}$.

\section{Deep-Inelastic Scattering}

Using the optical theorem and the Operator Product Expansion (OPE), the socalled Cornwall-Norton moments of the structure functions, that enter the hadronic tensor $W^{\mu \nu}$ for inclusive DIS on the nucleon, can be expanded as

$$
M_{n}\left(Q^{2}\right)=\int_{0}^{1} d x x^{n-2} F\left(x, Q^{2}\right)=\sum_{t} C_{n t}\left[\alpha_{s}\left(Q^{2}\right) \log \left(Q^{2}\right)\right] \frac{\mathcal{O}_{n}^{(t)}}{\left(Q^{2}\right)^{t / 2-1}},
$$

where the Wilson coefficients $C_{n t}$ are singular in the limit $Q^{2} \rightarrow 0$, while $\mathcal{O}_{n}^{(t)}$ are matrix elements of regular, symmetric, traceless local operators. The $C_{n t}$ can be calculated within pQCD at the given order $n$; they describe the loop corrections that accurately reproduce the observed logarithmic violations to scaling in the Bjorken variable $x$. The $\mathcal{O}_{n}^{(t)}$ contain information on the soft physics and are essentially unknown. They are related to nonperturbative power corrections through the expansion in twist powers $t$ of $1 / Q^{2}$, starting from the leading twist $t=2$. Both $C_{n t}$ and $\mathcal{O}_{n}^{(t)}$ implicitly depend on a renormalization and factorization scales (which will be understood in the following), but in any case the OPE gives an automatic and rigorous factorization between hard and soft scale physics, even if the proof is limited just to inclusive DIS (and $e^{+} e^{-}$annihilation).

The extraction of the socalled higher twists, which in parton language represent multiparton correlations, is of delicate importance in determining 
the full $Q^{2}$ dependence of the hadronic tensor in the preasymptotic region, since they affect accurate extraction from data of the leading-twist parton distributions, which are interpreted as parton matrix elements of one-body operators. In the next Sec. 3.1 the issue of higher twist extraction will be discussed for the unpolarized and polarized structure functions entering $W^{\mu \nu}$. In Sec. 3.2 the properties of leading-twist parton distributions will be described, with particular emphasys on the socalled transversity distribution, which, being a chiral odd object, is unreachable in inclusive DIS and represents the missing cornerstone for completing the knowledge of the leading-twist spin distribution of quarks inside a hadron. Finally, in Sec. 3.3 I will discuss the possibility of linking the parton distributions to the wave functions of constituent quarks by properly defining a nonperturbative, low-scale, phenomenological input to the Altarelli-Parisi evolution equations.

\subsection{Structure Functions and Higher Twists}

In Ref. ${ }^{19}$, the moments $M_{n}\left(Q^{2}\right)$ in Eq. (1) are obtained by fitting the world data for inclusive electron scattering on protons and deuterons in the DIS kinematics, including data from SLAC and BCDMS, over the range $1<Q^{2}<20(\mathrm{GeV} / c)^{2}$ and also for invariant masses lower than $2.5 \mathrm{GeV}$. Since for each $Q^{2}$ the whole range in $x$ is needed, it is necessary to use phenomenological fits that interpolate the existing data also in the unexplored kinematics and unavoidably introduce a certain amount of uncertainty. Since the OPE holds for inclusive reactions only, both elastic and inelastic contribution have been taken into account, the proton elastic ones being analytically calculated in terms of its form factors $G_{E}^{\mathrm{p}}, G_{M}^{\mathrm{p}}$, while the convolution formula with a realistic $\mathrm{N}$ momentum distribution is adopted for the deuteron. In Ref. ${ }^{19}$ it is also remarked that moments in the Nachtmann variable $\xi$ are preferable, since they allow to filter out of $M_{n}$ spurious higher-twist contributions coming from power-like target mass corrections due to the contributions from $\mathcal{O}_{n^{\prime}}^{(t)}$ operators with $n^{\prime} \neq n$.

The expansion on the right-hand side of Eq. (1) is approximated up to twist $t=6$ by using the calculated pQCD result at Next-to-Leading Order (NLO) for the leading twist $t=2$, and by introducing effective anomalous dimensions $\gamma_{n 4}, \gamma_{n 6}$ for the twists $t=4,6$, respectively, as input parameters. The NLO approximation to the leading twist can be further improved in two ways. A Sudakov form factor can be included that accounts for the Soft Gluon Radiation (SGR) at any order by using resummation techniques. 
The correction is required by the Wilson coefficients $C_{n 2}$ being divergent as $\log (n)$ for $x \rightarrow 1$, where the cancellation between singularities produced by virtual and real gluon loops is lost ${ }^{19}$. Alternatively, at the one-loop level there are power corrections deriving from vacuum polarization insertions in gluon lines, called Infra-Red (IR) renormalons, that cannot be resummed in Sudakov form factors; at low $Q^{2}$ they can simulate twist corrections, making the extraction of the latter rather ambiguous.

By fitting the Nachtmann moments on the left-hand side of Eq. (1) with the effective anomalous dimension approximation on the right-hand side, it is possible to constrain the discussed parameters and extract information on the higher twists. The main result is that there is a cancellation between the effective twist- 4 and twist- 6 terms, but each one individually is not negligible; this outcome still leaves open the question about a possible alternating sign in the twist expansion. It seems, anyway, crucial to simultaneously extract all the twists from data. In fact, a comparison with the corresponding analysis obtained by fitting the leading twist with a NLO parametrization from Glück, Reya and Vogt (GRV), indicates that small differences at leading twist reflect in larger ones at the much smaller higher twists. Most interestingly, the comparison between the calculated leadingtwist $M_{n}$ for the deuteron structure functions and the corresponding GRV parametrization suggests that the discrepancy of the latter with data can be eliminated by enhancing the ratio $d(x) / u(x)$ for the $u, d$ distributions in the region $x \rightarrow 1$, to which the higher $n$ moments are mostly sensitive ${ }^{19}$. This suggestion is consistent with the observed pQCD result $\frac{3}{7}$ for the ratio $F_{2}^{\mathrm{n}}(x) / F_{2}^{\mathrm{p}}(x)$ in the elastic limit, against an experimental (questioned) datum of $\frac{1}{4}$. IR renormalons overestimate the higher-twist contribution with respect to the SGR correction, which is preferable also because it is analytically calculable.

The above sketched procedure can be extended to inclusive polarized electron scattering to extract Nachtmann moments of the polarized structure functions. To this purpose, a new phenomenological parametrization of $G_{1}^{\mathrm{p}}\left(x, Q^{2}\right)$ has been developed ${ }^{20}$ by interpolating the world data on proton targets in the DIS regime for $x \gtrsim 0.02$ and $1<Q^{2}<50(\mathrm{GeV} / c)^{2}$, as well as the available results for photo- and electro-production of proton resonances. The parameters are constrained to reproduce the experimental value of the Gerasimov-Drell-Hearn (GDH) sum rule. Accordingly, the $Q^{2}$ dependent generalized GDH sum rule is predicted to have a zero-crossing point at $Q^{2}=0.16 \pm 0.04(\mathrm{GeV} / c)^{2}$. For the first moment, the higher twists are very small. For higher-order moments, particularly sensitive to 
the $x \rightarrow 1$ region, the SGR correction reduces the size of higher twists, which, however, remain still relevant for $Q^{2}$ of few $(\mathrm{GeV} / c)^{2}$, at variance with the unpolarized case. This suggests that spin-dependent multi-parton correlations could have a bigger impact than the spin-independent ones. Finally, comparing the trend in the Nachtmann variable $\xi$ of the interpolated data for $G_{1}^{\mathrm{p}}\left(\xi, Q^{2}\right)$ at different low values of $Q^{2}$ and at $Q^{2}=20(\mathrm{GeV} / c)^{2}$ in the DIS regime, an explicit violation of the Bloom-Gilman duality for polarized electroproduction of $\Delta$ on protons is reported.

\subsection{Parton Distribution and Fragmentation Functions}

Assuming factorization between hard and soft physics happening at very different scales, the hadronic tensor $W^{\mu \nu}$ for inclusive DIS on nucleons can be analyzed by the approach based on the diagrammatic expansion. The leading-order contribution, both in twist and powers of $\left(\alpha_{s} \log \left(Q^{2}\right)\right)$, is given by the well-known handbag diagram that involves, in the light-cone gauge $A^{+}=0$, the soft quark-quark correlation function

$$
\Phi_{a}(p)=\int d^{4} \xi e^{-i \xi \cdot p}\left\langle P, S\left|\bar{\psi}_{a}(\xi) \psi_{a}(0)\right| P, S\right\rangle,
$$

which describes the behaviour of a parton with flavor $a$ and momentum $p$ in a target hadron with momentum $P$ and spin $S$ through the hadronic matrix element of a leading-twist bilocal operator. Applying the Fierz decomposition to $\Phi_{a}(p)$, an expansion in terms of projections $\Phi_{a}^{\Gamma}(x)$ onto a complete set of Dirac structures $\Gamma$ is obtained, where $x=p^{+} / P^{+}$is the light-cone momentum fraction of the parton inside the parent hadron for the dominant light-cone direction "+" induced by the electromagnetic interaction (at the considered leading order, the difference between $x$ and Bjorken variable will be consistently neglected). The $\Gamma=\gamma^{+}, \gamma^{+} \gamma_{5}, i \sigma^{i+} \gamma_{5}$ projections give the leading-twist parton model distribution functions: the momentum distribution $f_{1}(x)$, the helicity distribution $g_{1}(x)$ and the transversity distribution $h_{1}(x)$. Therefore, while three distributions are needed to reach a complete knowledge of the leading-twist momentum and spin structure of the quarks with respect to a preferred longitudinal direction, only two of them, $f_{1}$ and $g_{1}$, have been extensively studied through measurements of the corresponding unpolarized and longitudinally polarized structure functions, respectively. The reason is that the transversity $h_{1}$ is related to helicity flipping mechanisms; hence, it is a chiral-odd object and requires the occurrence of another chiral-odd partner in any observable to be measured. In inclusive DIS at leading twist, there are no mechanisms providing 
such partners because chirality is preserved in hard pQCD. Nevertheless, $h_{1}$ is a very interesting object. I forward the interested reader to the very comprehensive report of Ref. ${ }^{21}$ on the $h_{1}$ properties. Here, it is sufficient to mention that its integral measures the tensor charge, whose determination would give access to chiral-odd operators in QCD and, consequently, to the role of chiral symmetry in the hadron structure. Because of the mismatch in helicities, $h_{1}$ has a non-singlet evolution disconnected from gluons, hence more smooth than the one of $g_{1}$. In the hadron rest frame, $h_{1}$ and $g_{1}$ would be perfectly equivalent because of the rotational symmetry of the system. However, the distributions are defined after the system is boosted along a preferred longitudinal direction induced by the electromagnetic interaction, thus breaking the rotational invariance. Therefore, the difference between $h_{1}$ and $g_{1}$ tells us also about the relativistic nature of the dynamics of quarks inside the hadron.

Originally, extraction of $h_{1}$ was proposed by measuring spin asymmetries in Drell-Yan reactions with two colliding transversely polarized protons; however, even if the process occurs at leading twist, the probability of finding transversely polarized antiquarks is presumable much smaller than for quarks. In DIS, semi-inclusive processes need to be considered to provide the necessary chiral-odd partner through the quark hadronization into one or several detected hadrons. Similarly to Eq. (2), the corresponding soft correlation function can be parametrized in terms of the socalled fragmentation functions, which also have at leading twist a probabilistic interpretation and are interesting quantities by themselves since they bear witness of how confinement arises. However, the twist analysis reveals that for detected spinless hadrons chiral-odd fragmentation functions appear only at the twist-three level. The price to pay for having such objects at leading twist is to detect baryons, such as the $\Lambda$ particle, in socalled double-spin asymmetries where both the target and the $\Lambda$ are polarized. Before commenting on this option, I will discuss the simpler alternative of the single-spin asymmetry, where only the target is polarized and final mesons (basically pions) are abundantly measured in the detector. The key observation is that if memory of the transverse quark dynamics is kept inside the various distributions, it is possible to build an azimuthal asymmetry related to the socalled Collins angle $\sin \phi_{C} \propto \mathbf{P}_{h} \times \mathbf{k} \cdot \mathbf{S}_{T}$, where $P_{h}$ is the detected hadron momentum with a nonvanishing transverse component with respect to the jet axis and $\mathbf{k}$ is the fragmenting quark momentum with transverse polarization $\mathbf{S}_{T}{ }^{22}$. By properly weighting the single-spin asymmetry obtained by flipping the transverse polarization of a nucleon target, 
it is possible to isolate $h_{1}$ at leading twist by means of the first moment of the socalled Collins function $H_{1}^{\perp}\left(z, \mathbf{k}_{T}\right)$, where $z=P_{h}^{-} / k^{-}$is the lightcone momentum fraction of the fragmenting quark carried by the detected hadron. This chiral-odd fragmentation function is given by the probability difference of quarks with opposite transverse polarization to fragment into an unpolarized hadron; it can be interpreted as a sort of analyzing power of the quark polarization into the orbital angular motion of the detected hadron.

The Collins effect has been measured at Fermilab using the reaction $p p^{\uparrow} \rightarrow \pi X$ and by the HERMES collaboration using the DIS $e \vec{p} \rightarrow e^{\prime} \pi X$, where the longitudinal polarization of the target along the beam direction has a small transverse component with respect to the direction of the virtual photon ${ }^{23}$. Because of such an effective small polarization and of an asymmetry dilution due to NLO corrections introduced by SGR techniques, it came recently as a surprise that the measured asymmetry for all detected isospin states of the pion has a remarkable absolute size of as much as $5 \%$. At present, there is an ongoing discussion about the issue if factorization is under control or if other possible mechanisms could generate a nonvanishing single-spin asymmetry that could make the $h_{1}$ extraction questionable. I refer the interested reader to Ref. ${ }^{24}$ and references therein. Here, I will just mention the work of Ref. ${ }^{25}$, where the Weinberg's idea of a generalized time-reversal operator is applied to a flavor multiplet of quarks interacting with chiral fields. By mixing up the flavors inside the multiplet on top of the usual time-reversal transformation, the internal $\mathrm{N}$ chiral dynamics generates $\mathbf{k}_{T}$-dependent distributions functions that would be forbidden by the standard time-reversal operator, but are perfectly legitimate under the new nonstandard one. This would open the possibility of a larger class of transverse single-spin asymmetries. Because of the supposed universality of such naive $T$-odd distributions, the same possibility should be open also in semi-inclusive lepton DIS, thus giving a possible interpretation of the HERMES data.

A very promising alternative to the Collins effect is represented by the single-spin asymmetry generated by the correlation between the transverse polarization of the fragmenting quark and the orientation of a $\pi^{+} \pi^{-}$pair inside the jet in semi-inclusive production of two leading pions. The effect is proportional to the azimuthal angle $\sin \phi \propto \mathbf{P}_{\pi^{+}} \times \mathbf{P}_{\pi^{-}} \cdot \mathbf{S}_{T}$ and can be interpreted as due to the interference between different relative partial waves of the pion pair with different phases. By generalizing the soft correlation function to this case, a leading-twist analysis reveals that a new class of 
chiral-odd (and naive $T$-odd) fragmentation functions arises, the socalled Interference Fragmentation Functions (IFF), that depend in a complicated way upon the kinematics and the "geometry" of the reaction ${ }^{26}$. However, in DIS a single-spin asymmetry can isolate $h_{1}$ at leading twist through $H_{1}^{\varangle}$, which is an IFF sensitive to the transverse component of the pair relative momentum ${ }^{27}$. The asymmetry is based on a collinear factorization theorem, where the $\mathbf{k}_{T}$ dependence can be integrated away leading to a cancellation of soft gluon radiation effect, at variance with the Collins function; an analysis beyond tree level seems, therefore, straightforward. The $H_{1}^{\varangle}$ could be extracted, in principle, from the corresponding $e^{+} e^{-}$annihilation. At present, its knowledge relies upon models. In the context of the spectator model approximation, the single-spin asymmetry for the DIS $e p^{\uparrow} \rightarrow e^{\prime}\left(\pi^{+} \pi^{-}\right) X$ has been computed by modelling the IFF as originating from the interference between an $s$-wave direct $\pi \pi$ production and a $p$-wave decay of the $\rho$ resonance, leading to an asymmetry as large as $1.5 \%{ }^{27}$.

I will now go back to the case of semi-inclusive production of the $\Lambda$ particle. For the semi-inclusive production of transversely polarized $\Lambda^{\uparrow}$, a double-spin asymmetry depends at leading twist upon the product $h_{1} H_{1}$, where $H_{1}$ is a chiral-odd (naive $T$-even) fragmentation function related to the probability of a transversely polarized quark to fragment into a transversely polarized baryon. A sizeable asymmetry has been observed at Fermilab, but the low values of $\Lambda^{\uparrow}$ transverse momentum explored prevent from using any factorization in the analysis of the cross section, thus posing questions on the $h_{1}$ extraction from these data. Moreover, modelling $H_{1}$ is a challenge since the mechanism for the polarization transfer from the quark to the $\Lambda$ is still unknown. The data collected at Fermilab for the unpolarized $\Lambda^{\uparrow}$ production $\left(p p \rightarrow \Lambda^{\uparrow} X\right)$ also show a very large asymmetry around $20 \%$, while pQCD predicts an almost vanishing effect if the process is assumed collinear. Since the $\Lambda$ polarization seems roughly independent from the target, a possible interpretation is given by a new nonperturbative spin effect that produces an asymmetry proportional to the azimuthal angle $\sin \phi \propto \mathbf{k} \times \mathbf{k}_{T} \cdot \mathbf{S}_{\Lambda}$. It is parametrized by the polarized Fragmentation Function (polFF) $D_{1 T}^{\perp}$, which is related to the probability of an unpolarized quark to fragment into a transversely polarized hadron ${ }^{28}$. The $D_{1 T}^{\perp}$ is naive $T$-odd but chiral even; therefore, it can be easily detected also in $\Lambda$ productions with charged-current processes. Assuming a factorization theorem and charge conjugation invariance to hold, a fit to the data for the $p \mathrm{Be} \rightarrow \Lambda^{\uparrow} X$ and $p \mathrm{Be} \rightarrow \bar{\Lambda}^{\uparrow} X$ can be achieved with a simple parametrization for $D_{1 T}^{\perp}$. The known trends of a large negative $\Lambda$ polarization, increas- 
ing with $x_{F}=p_{\Lambda} / p_{\text {beam }}$, of its puzzling flat dependence on $\Lambda$ transverse momenta beyond $1 \mathrm{GeV} / c$, and of an almost vanishing $\bar{\Lambda}$ polarization, are reasonably reproduced ${ }^{28}$. However, the Fermilab data again prevent any interpretation in terms of factorization. Therefore, the extracted $D_{1 T}^{\perp}$ cannot be used in the $e p \rightarrow e^{\prime} \Lambda^{\uparrow} X$ reaction, where only speculations based on different scenarios and different choices of parameters are possible, at present ${ }^{29}$.

When the $\Lambda$ and $\bar{\Lambda}$ are longitudinally polarized, new possibilities are available for deepening the knowledge of parton distributions ${ }^{30}$. Working at LO in pQCD, the cross section for the process $\overrightarrow{l N} \rightarrow l^{\prime} \vec{\Lambda} X$ has eight helicity components which can be reduced to just four by $P$-invariance and helicity conservation. Assuming charge-conjugation and isospin invariance, linear combinations of these four components for $\mathrm{p}$ and $\mathrm{n}$ targets allow to extract information on the polarized strange quark density $g_{1}^{s}(x)+g_{1}^{\bar{s}}(x)$ and on the validity of the hypothesis $f_{1}^{s}(x)=f_{1}^{\bar{s}}(x)$ and $g_{1}^{s}(x)=g_{1}^{\bar{s}}(x)$, that are not reachable in unpolarized DIS ${ }^{31}$. By generalizing the analysis also to charge-current processes and $e^{+} e^{-}$annihilations leading to $\vec{\Lambda}$ and $\vec{\Lambda}$, various parametrizations can be considered for the unpolarized $D_{1}^{a}$ and longitudinally polarized $G_{1}^{a}$ fragmentation functions for quark flavor $a$ to fit recent data from various laboratories ${ }^{32}$, in order to test various scenarios according to different assumptions in the flavor SU(3) decomposition.

\subsection{From Constituent Quarks to Parton Distributions}

It has already been remarked in Sec. 3.1 that the ratio $F_{2}^{\mathrm{n}}(x) / F_{2}^{\mathrm{p}}(x)$ in the limit $x \rightarrow 1$ is an observable very sensitive to the symmetry properties of the underlying theory, which can influence the parton distributions, in particular the ratio $d(x) / u(x)$. When studying the quark distributions, usually, the second moment of the valence quark distribution is evolved back from a low-energy parametrization to a low-energy scale $\mu_{0}$ where the short-range perturbative contribution (related to the gluon and sea quarks) is negligible and the valence distribution becomes dominant. Then, $\mathrm{LO}$ or NLO evolution is applied up to the selected experimental scale and the structure functions are deduced in the partonic description of quark distributions. Since the parton model emerges in the Bjorken limit because of light-cone dominance, it can be developed in the hadron rest frame using the light-cone formalism. The low-energy parametrization of the polarized valence quark distribution $q_{\lambda}^{\mathrm{val}}\left(x, \mu_{0}^{2}\right)$ can then be replaced by the light-cone momentum density $n_{\lambda}$ of the parton with helicity $\lambda$. The latter can be 
calculated in the light-front formulation of a relativistic interacting threebody sistem (see Sec. 2.1). Since, moreover, the usual scale $\mu_{0}$ turns out to be very close to the constituent quark mass, the partons in the hadron rest frame can be identified with the constituent quarks. Thus, the lowenergy input to the evolution equations can be related to the CQM N wave functions, that are deduced by satisfying covariance and the Pauli principle. In this way, it is possible to test at the high DIS scale the mechanisms proposed for $\mathrm{SU}(6)$ breaking at low scale. The issue has been debated since long time, but only recently in Ref. ${ }^{33}$ a correct treatment of the effects related to covariance and Pauli principle have been accounted for. For the range $0.3 \lesssim x \lesssim 0.7$, where the valence quark picture dominates and the approach is meaningful, the ratio $F_{2}^{\mathrm{n}}(x) / F_{2}^{\mathrm{p}}(x)$ has been investigated using the two potentials $V_{\mathrm{OGE}}$ and $V_{\mathrm{GBE}}$. Both interactions give asymptotic values different from the $\mathrm{SU}(6)$ result $\frac{2}{3}$, but fail to reproduce the high- $x$ trend of the CTEQ5 parametrization fitting the data, even if the ratio shows a rather weak sensitivity to QCD radiative effects introduced by evolution. The disagreement emerges only if the effects due to Pauli principle are properly included ${ }^{33}$.

The same technique can be applied to the study of the orbital angular momentum of the hadron constituents ${ }^{34}$. The interest in this observable is dictated by the common belief that the $\mathrm{N}$ total angular momentum of $\frac{1}{2}$ must be shared among its constituents as $\frac{1}{2}=\frac{1}{2} \Delta \Sigma+\Delta g+L_{q}+L_{g}$, where $\Delta \Sigma, \Delta g$ are the helicities of quarks and gluons, respectively, and $L_{q}, L_{g}$ are the quark and gluon orbital angular momenta. A gauge invariant definition of $L_{q}$ and $L_{g}$ involves the socalled Generalized Parton Distributions (GPD), that will be addressed in the next Sec. 4. Here, a low-energy model calculation of $L_{q}$ and $\Delta \Sigma$ is obtained by calculating matrix elements of the $\sum_{i}-i\left(\mathbf{k}_{i} \times \nabla_{i}\right)$ and $\left(n_{\lambda}-n_{-\lambda}\right)$ operators on $\mathrm{SU}(6)$ wave functions obtained in LF description of the hypercentral CQM described in Sec. $2^{34}$. Melosh rotations of the quark spins generate a nonvanishing $L_{q}$ at the input scale $\mu_{0}$ even if the $\mathrm{N}$ state is in a $S$ wave. The model verifies the $\mathrm{N}$ angular momentum sum rule, which reads $\frac{1}{2} \Delta \Sigma\left(\mu_{0}^{2}\right)+L_{q}\left(\mu_{0}^{2}\right)=0.228+0.272=0.5$. Then, evolution mixes up the two contributions, but the effect of Melosh rotations and of high momentum components, generated by $V_{\text {hyp }}$, survives at large $x$. Evolution produces also $L_{g}\left(Q^{2}\right) \neq 0$, even if $L_{g}\left(\mu_{0}^{2}\right)=0$. The values obtained in the range $1<Q^{2}<20(\mathrm{GeV} / c)^{2}$ are compatible with the ones obtained by QCD sum rules and lattice calculations ${ }^{34}$. 


\section{Generalized Parton Distributions}

As already anticipated in the previous subsection, Generalized Parton Distributions (GPD) represent a possible tool to address the orbital angular momentum of quarks inside hadrons in a gauge-invariant way. Intuitively, GPD are recovered by considering the handbag diagram of inclusive DIS and pushing it to the off-diagonal components in the hadron and parton legs, as it may happen for the leading-order diagram of Deeply Virtual Compton Scattering (DVCS) or of hard exclusive Meson Production (DVMP). For very large $Q^{2}$ of the entering virtual photon, for large $\mathrm{cm}$ energy $s$ and small momentum transfer $t$, a factorization theorem can be rigorously proven between the hard electromagnetic interaction and the soft physics parametrized in terms of a quark-quark correlation function, that generalized the one in Eq. (2). The latter can be expanded in the relevant Dirac structure displaying, at leading twist, two unpolarized and two polarized helicity-conserving and helicity-flipping GPD. Because of the factorization theorem, the GPD formalism represents a unifying framework for whole classes of inclusive and exclusive processes. In fact, in the forward limit the usual parton distributions are recovered. The first moments in $x$ produce the electromagnetic form factors $F_{1}(t), F_{2}(t)$; if the final hadrons are $\mathrm{N}$ resonances, the transition form factors are also obtained. From GPD spatial transverse distributions can be defined that give information on the localization of partons inside hadrons. Finally, at $t=0$ the $m$-th moments in $x$ are even polynomials of degree $m$ in the skewedness parameter $\xi$, which is related to the mismatch in the longitudinal hadron momentum. This constraint, called polynomiality property, is a very general one, since it follows from Lorentz invariance. When applied to the unpolarized GPD for $m=2$, a gauge invariant definition of the quark total angular momentum is obtained.

The experimental extraction of GPD is a very challenging task. Recently, data have been obtained for the beam spin and charge asymmetry, and for DVCS, at the DESY and TJNAF laboratories; these measurements, however, allow for mapping GPD only in a very limited part of the phase space. Therefore, at present their understanding relies mostly on models. In the literature, there are basically two different approaches. The first one is a phenomenological construction based on a reduction formula where the GPD are related to the parton distributions by factorizing the $t$ dependence due to the electromagnetic form factors. The latter approach is based on a direct calculation with specific dynamical assumptions. In Ref. ${ }^{35}$, the chi- 
ral quark-soliton model is considered, based on the instanton realization of the nonperturbative QCD vacuum, and a further proof of its internal consistency is given by analytically checking that the polynomiality property is satisfied. As a byproduct, explicit expressions are obtained for all the coefficients of the $m$-th order polynomial; in particular, the highest-order coefficient gives the $m$-th moment of the socalled $D$-term, which gives the behaviour of the GPD in the nonvalence domain $-\xi<x<\xi$.

By expanding the $\mathrm{N}$ state upon valence light-cone wave functions in the Fock space for three partons, the off-diagonal soft correlation function can be represented as an overlap of the valence light-cone wave functions themselves. Very similarly to Sec. 3.3, the GPD can then be represented in terms of the off-diagonal light-cone density matrix, which, in turn, can be calculated by solving the eigenvalue problem for some quark-quark potential in a CQM and then by transforming the solutions to the light-cone via the LF boosts ${ }^{36}$. The correct covariance of the approach grants the good properties of the GPD, namely recovering the parton distributions with the correct support in the forward limit and automatically fulfilling the particle number and momentum sum rules. However, since the GPD are calculated from valence light-cone wave functions, they are defined only in the region $\xi \leq x \leq 1$; therefore, their first moment gives the $\mathrm{N}$ electromagnetic form factors only in the limit $\xi \rightarrow 0$. In Ref. ${ }^{36}$, the leading-twist unpolarized GPD are calculated starting from the $V_{\text {hyp }}$ and $V_{\text {GBE }}$ potentials described in Sec. 2. No particular difference is obtained with the two choices, despite the fact the former is $\mathrm{SU}(6)$ symmetric and the latter contains SU(6)-breaking terms. Remarkably, the unpolarized helicity-flipping GPD is completely determined by the effect of Melosh rotations in boosting the CQM wave functions on the light-cone. A strong $t$ and a weak $\xi$ dependence are observed.

\section{References}

1. M. De Sanctis, M.M. Giannini, L. Repetto and E. Santopinto, Phys. Rev. C62, 025208 (2000).

2. See M. De Sanctis, these proceedings.

3. M.M. Giannini, E. Santopinto, and A. Vassallo, Eur. Phys. J. A12, 447 (2001).

4. G. Dillon and G. Morpurgo, Phys. Lett. B481, 239 (2000).

5. G. Dillon and G. Morpurgo, EuroPhys. Lett. 54, 35 (2001).

6. G. Dillon and G. Morpurgo, hep-ph/0011202.

7. G. Morpurgo, hep-ph/0107049.

8. E. Pace, G. Salmè, F. Cardarelli, and S. Simula, Nucl. Phys. A666-667, 33 (2000). 
9. F.M. Lev, E. Pace, and G. Salmè, Phys. Rev. C62, 064004 (2000); Nucl. Phys. A663-664, 365 (2000).

10. F. Cardarelli and S. Simula, Phys. Rev. C62, 065201 (2000).

11. S. Simula, Proceedings of the International Conference The Physics of the Excited Nucleon ( $N^{*}$ 2001), Mainz, March 2001, hep-ph/0105024.

12. D. Melikhov and S. Simula, Phys. Rev. D65, 094043 (2002).

13. S. Simula, Phys. Rev. C66, 035201 (2002).

14. J.P.B.C. de Melo, T. Frederico, E. Pace, and G. Salmè, Nucl. Phys. A707, 399 (2002).

15. R.F. Wagenbrunn, S. Boffi, W. Klink, W. Plessas, and M. Radici, Phys. Lett. B511, 33 (2001).

16. L. Glozman, M. Radici, R.F. Wagenbrunn, S. Boffi, W. Klink, and W. Plessas, Phys. Lett. B516, 183 (2001).

17. S. Boffi, L. Glozman, W. Klink, W. Plessas, M. Radici, and R.F. Wagenbrunn, Eur. Phys. J A14, 17 (2002).

18. W.M. Alberico, F. Giacosa, M. Nardi, and C. Ratti, Eur. Phys. J. A, in press, nucl-th/0206071.

19. G. Ricco, S. Simula, and M. Battaglieri, Nucl. Phys. B555, 306 (1999); S. Simula, Phys. Lett. B493, 325 (2000).

20. S. Simula, M. Osipenko, G. Ricco, and M. Taiuti, Phys. Rev. D65, 034017 (2002).

21. V. Barone, A. Drago, and P.G. Ratcliffe, Phys. Rept. 359, 1 (2002).

22. M. Anselmino, M. Boglione, J. Hansson, and F. Murgia, Eur. Phys. J. C13, 519 (2000).

23. M. Anselmino and F. Murgia, Phys. Lett. B483, 74 (2000).

24. F. Murgia, these proceedings.

25. M. Anselmino, V. Barone, A. Drago, and F. Murgia, hep-ph/0209073.

26. A. Bianconi, S. Boffi, R. Jakob, and M. Radici, Phys. Rev. D62, 034008 (2000); ibidem, 034009 (2000).

27. M. Radici, R. Jakob, and A. Bianconi, Phys. Rev. D65, 074031 (2002).

28. M. Anselmino, D. Boer, U. D'Alesio, and F. Murgia, Phys. Rev. D63, 054029 (2001).

29. M. Anselmino, D. Boer, U. D'Alesio, and F. Murgia, Phys. Rev. D65, 114014 (2002).

30. M. Anselmino, M. Boglione, U. D'Alesio, and F. Murgia, Eur. Phys. J. C21, 501 (2001).

31. M. Anselmino, M. Boglione, U. D'Alesio, E. Leader, and F. Murgia, Phys. Lett. B509, 246 (2001).

32. M. Anselmino, M. Boglione, and F. Murgia, Phys. Lett. B481, 253 (2000).

33. B. Pasquini, M. Traini, and S. Boffi, Phys. Rev. D65 074028 (2002).

34. F. Cano, P. Faccioli, S. Scopetta, and M. Traini, Phys. Rev. D62, 054023 (2000).

35. P. Schweitzer, S. Boffi, and M. Radici, Phys. Rev. D66, in press, hep-ph/0207230.

36. S. Boffi, B. Pasquini, and M. Traini, hep-ph/0207340. 